MATEC Web of Conferences 4, 01004 (2013)

DOI: $10.1051 /$ matecconf/20130401004

(C) Owned by the authors, published by EDP Sciences, 2013

\title{
Fabrication of semi-transparent superoleophobic thin film from fabrics and nanoparticle-based hierarchical structure
}

\author{
S. Nishizawa ${ }^{1}$ and S. Shiratori ${ }^{1}$ \\ ${ }^{1}$ Graduate School of Science and Technology, Keio University, Japan \\ Email: shiratori@appi.keio.ac.jp
}

\begin{abstract}
Superoleophobic thin films have many potential applications including fluid transfer, fluid power systems, stain resistant and antifouling materials, and microfluidics among others. Transparency is also desired with superhydrophobicity for their numerous applications; however transparency and oleophobicity are almost incompatible relationship with each other in the point of surface structure. Because oleophobicity required rougher structure at nano-micro scale than hydrophobicity, and these rough structure brings light scattering. So far, there is very few report of the compatible of transparency and superoleophobicity. In this report, we proposed the see-through type fabrics using the nanoparticle-based hierarchical structure thin film for improving both of oleophobicity and transparency. The vacant space between fibrils of fabrics has two important roles: the one is to through the light, another one is to introduce air layer to realize Cassie state of liquid droplet on thin film. To realize the low surface energy and nanoscale rough structure surface on fibrils, we used the spray method with perfluoroalkyl methacrylic copolymer (PMC), silica nano particles and volatile solvent. From the SEM image, the hierarchical structures of nanoparticle were formed uniformly on the fabrics. The transparency of thin film obtained was approximately $61 \%$ and the change of transparency between pre-coated fabrics and coated was $11 \%$. From investigation of the surface wettability, the contact angles of oils (rapeseed oil and hexadecane) and water droplet on the fabricated film were over 150 degree.
\end{abstract}

\section{Introduction}

Extreme wetting behavior, namely super-repellency and super- wetting, has recently received enormous commercial and academic interest because of its wide applicability in various fields including self-cleaning surfaces [1-3], microfluidic channels, and anti-fogging films [2]. Surface wettability is basically described by the Cassie [4] and Wenzel [5] theories, which have emerged from surface energy and surface roughness. Extremely wettable surfaces are realized by controlling the wettability through surface energy and surface roughness of thin films.

Superoleophobic coatings have many potential applications including fluid transfer, fluid power systems, stain resistant and antifouling materials, and microfluidics [6-8]. The mechanism of superoleophobic films is based on the theory of wettability used to explain hydrophobicity, and this function is realized by achieving low surface energy and high surface roughness. Because the surface tension of oils is lower than that of water, the fabrication of superoleophobic surfaces is difficult. Especially, in many reports, the superoleophobic surface requires the hierarchical structure which contains nanomicro scale roughness.

Transparency is also desired with superhydrophobicity for their numerous applications; however transparency and oleophobicity are incompatible relationship with each other in the point of surface structure. As for the superhydrophobicity, there are some reports of superhydrophobic transparent films: the required surface roughness for it is under hundred nano meters. [9-10]. Otherwise, high oleophobicity requires rough surface structure in micro scale, thus it causes light scattering, and as a consequence, the transparency of thin film decreases drastically. So far, there are few reports that contain both transparency and superoleophobicity [11-12].

On the other hand, in the study of conductive transparent film, relationship between transparency and conductivity is also incompatible in the high conductive level. As the approach for improving the conductivity and also transparency, the see-through type structure has 
recently attracted many interests for its possibility of improving both of conductivity and transparency [13-14]. Although the see-through type approach also scattered the light, the scattered ratio and space ratio can be controlled by its scale. Likewise transparent conductive films with see-through structure, the transparent superwettability thin film should be able to be designed.

In this report, we fabricated the see-through type fabrics with the nanoparticle-based hierarchical structure in order to ensure the compatibility between oleophobicity and transparency. This approach will be an efficient way for keeping liquids in Cassie-state which is significant for realizing superoleophobicity. The vacant space between fibrils of fabrics has two important roles: the one is to through the light, another is to induce air layer to realize Cassie state of liquid droplet on thin film. There are some approaches to fabricate superoleohobic thin films by using fabrics as substrate $[6,15]$. These reports mentioned about relationship of wettability and fabric structure, however, not about transparency nor the possibility of superoleophobic transparent films by fabrics based substrate. In this paper, we investigated the possibility of the superoleophobic transparent thin film with see-through fabric and $\mathrm{SiO}_{2}$ nanoparticle hierarchical structure.

\section{Experimental procedure}

\subsection{Materials}

See-through fabrics were obtained from Nisikawa Industry (Toyama, Japan). Diameters of each fibril are the $27 \mu \mathrm{m}$, and distances between fibrils are $150 \mu \mathrm{m}$, and the parallel transparency is $72.8 \%$. $\mathrm{SiO}_{2}$ nanoparticles (OX50, average primary particle diameter of $40 \mathrm{~nm}$ ) were obtained from Aerosil (Tokyo, Japan). Perfluoroalkyl methacrylic copolymer (PMC, Zonyl, dispersed in water $20 \mathrm{wt} \%)$ was obtained from Dupont. Distilled water $\left(\gamma_{\mathrm{lv}}\right.$ $\left.=72.8 \mathrm{mNm}^{-1}\right)$, rapeseed oil $\left(\gamma_{\mathrm{lv}}=33.0 \mathrm{mNm}^{-1}\right)$ and hexadecane $\left(\gamma_{l_{v}}=27.3 \mathrm{mNm}^{-1}\right)$ were used as probe liquids to evaluate contact angles. Distilled deionized water $\left(\gamma_{\mathrm{lv}}=72.8 \mathrm{mNm}^{-1}\right)$ with a resistance of 18.2 $\mathrm{M} \Omega . \mathrm{cm}$ was used as the solvent and as a probe liquid to evaluate contact angles.

\subsection{Fabrication of semi-transparent oleophobic film from spray coating}

At first, the $4.0 \mathrm{~g}$ of PMC was added into the $36.0 \mathrm{~g}$ of acetone. Then, the mixed solution was vigorous stirred and the bubble floated on the surface of solution was removed. Next, the certain amount of the PMC solution was moved into the vial bottle, and the $\mathrm{SiO}_{2}$ nanoparticle was added into it and the solution was stirred. The total weights of sol solutions were adjusted to $10 \mathrm{~g}$.

To improve the hydrophilicity of fabrics, the seethrough fabrics were plasma treated before spray coating. After this treatment, the fabrics were attached on the glass slide loosely with clips. And then the $10 \mathrm{~g}$ of $\mathrm{SiO}_{2} / \mathrm{PMC}$ solution were sprayed onto the fabrics with spray gun. After spraying, the fabrics were browed by air and dried at room temperature.

\subsection{Characterization}

Characterization of wettability: a commercial contact angle system (FACE, Kyowa Interface Science Co., Ltd, Japan) was used to measure the contact and roll-off angles of the thin films at room temperature. Water, rapeseed oil and hexadecane were used as probe liquids. The reported contact and roll-off angles are the average of measurements obtained at five different points on each sample surface. The measurement of static contact angles was performed using droplets of liquid with a diameter of $2 \mathrm{~mm}$.

Field emission scanning electron microscopy (FESEM) was performed on a Philips XL-30 ESEM FEG microscope (Philips, The Netherlands) in high-vacuum mode at an acceleration voltage of $5 \mathrm{kV}$. The transparencies of fabrics were measured by Haze meter (NDH-5000, Nippon Denshoku.co, Japan). We measured each samples at five times in different points of same samples. The optical image of water and oil droplets on the fabricated film was obtained by the digital camera (CX1, RIKO, Japan).

\section{Results and discussions}

\subsection{Surface structure of superoleophobic thin films}
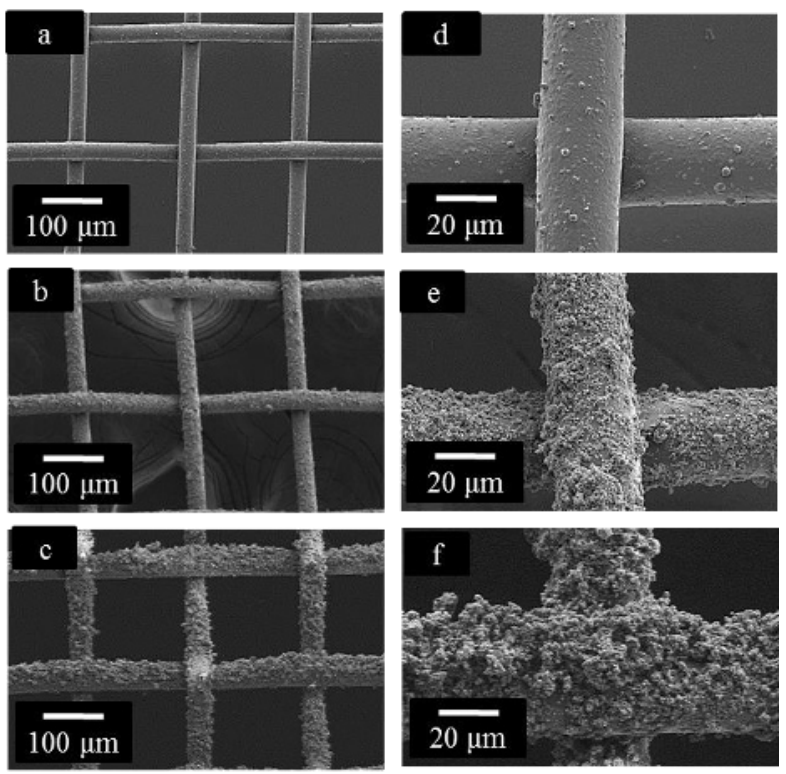

Figure 1. SEM images of spray-coated fabrics: each $\mathrm{SiO}_{2}$ particle ratios are $0 \mathrm{wt} \%$ ((a) and (d)), 0.5wt \% ((b) and (e)) and 1.5 wt \% ((c) and (f)).

Figure 1 shows SEM images and photo image of thin film obtained from spray-coating to the fabrics. The spray coating with see-through fabrics and volatile solvent succeeded to obtain the uniform nanoparticle coating on each fibril. These uniform structures were formed by the flexibility of fabrics and air blowing through spray 
process. From $0 \mathrm{wt} \%$ to $0.5 \mathrm{wt} \%$, the first layers were coated on the fibril and increase the surface roughness with the concentration of nanoparticle proportionally. At the concentration of $1.5 \mathrm{wt} \%$, the hierarchical structure was constructed on the fibrils. Therefore, this condition had the broad scale roughness not only fabrics' micro roughness but also aggregated particles' nano-micro roughness.

\subsection{Wettability of thin films}

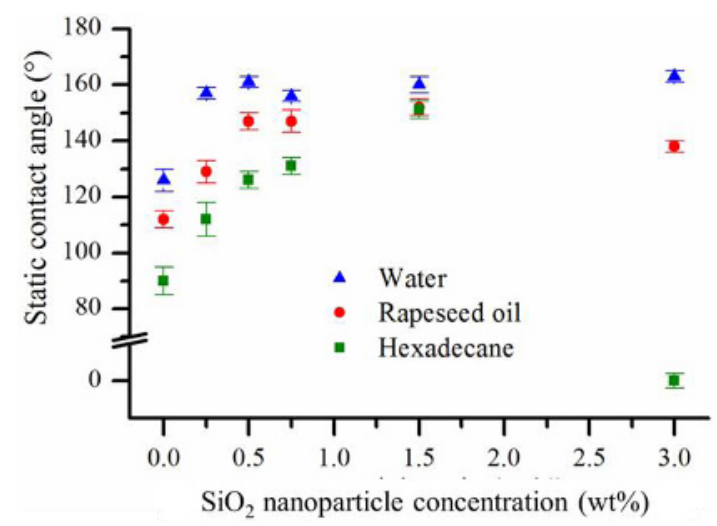

Figure 2. Contact angles of spray-coated tabrics against water and oils droplets

Figure 2 shows the surface wettability of spray-coated fabrics with water and oils droplets. Without $\mathrm{SiO}_{2}$ nanoparticle, the spray-coated fabrics showed hydrophobicity but not superhydrophobicity. By addition of the $\mathrm{SiO}_{2}$ nanoparticle, the hydrophobicity and also oleophobicity of fabrics were enhanced and they showed superhydrophobicity. As the $\mathrm{SiO}_{2}$ nanoparticle concentration increased from 0 wt $\%$ to $1.5 \mathrm{wt} \%$, the contact angles of droplets, especially low surface tension oils are enhanced. At the $\mathrm{SiO}_{2}$ nanoparticle concentration was 1.5 wt $\%$, the spray-coated fabric showed superoleophobicity against hexadecane. Despite the 3 wt $\%$ condition showed superhydrophobicity, the contact angle of hexadecane was 0 degree. We consider that this is because fluoride group of PMC cannot cover the surface of $\mathrm{SiO}_{2}$ particles completely when the PMC concentration was relatively lower than that of $\mathrm{SiO}_{2}$ particles, and this caused the surface superhydrophobicity and oleophilicity. According to the paper reported by Ming et.al. [15], multiple scale roughness from nanomicro inorganic particles on fabrics enhances oleophobicity. To discuss the wettability from multi scale surface roughness, they quoted the relationship between surface roughness and oleophobicity as follows:

$$
\cos \theta_{c^{*}}=r_{f} f \cos \theta_{Y}+f-1
$$

where $f$ is the area fraction of the solid-liquid interface, $r_{f}$ is the surface roughness factor of the wetted area, $\theta_{c^{*}}$ is the Cassie-state contact angle and $\theta_{0}$ is the Young contact angle of flat film. Especially, in the condition for contact angle of oil, which is smaller than $90^{\circ}$ against every surface, this above equation means the small contact area of the solid-liquid interface significantly enhances oleophobicity. In our research, the space between fibrils leads air layer into solid-liquid interface, and also the hierarchical structure from $\mathrm{SiO}_{2}$ nanoparticle on fibrils formed fine air layer into solid-liquid interface from its porous structure.

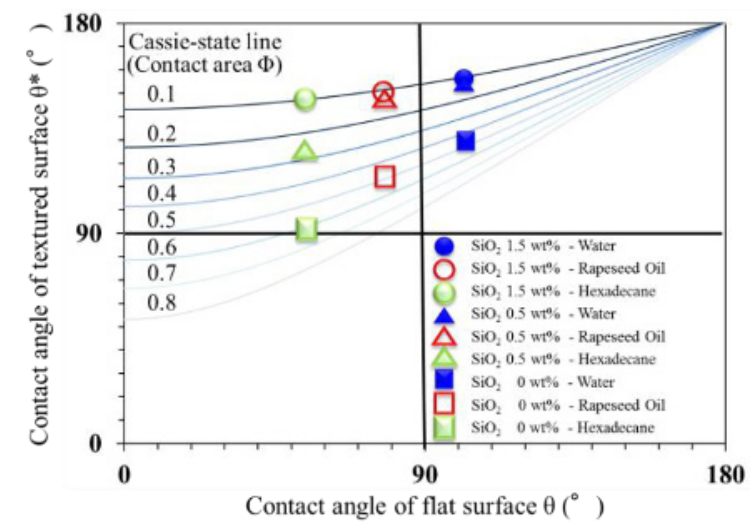

Figure 3. The application the Cassie-Baxter model to contact angles of flat surface and textured surface

The relationship of the contact angles of textured surface and flat surface is shown in Figure 3. In this figure, the Cassie state lines were drawn by changing the function of surface contact area with Cassies-Baxtar's equation [4] as follows:

$$
\cos \theta_{c^{*}}=f\left(1+\cos _{\theta Y}\right)-1
$$

where $f$ is the area fraction of the solid-liquid interface and $\theta_{c^{*}}$ is the Cassie-state contact angle and $\theta_{0}$ is the contact angle of flat surface. If we consider the Cassie state curves, the contact angles of spray-coated fabrics and flat surface, it is clearly demonstrated that all spraycoated fabrics maintain the Cassie state. However, the low concentration $\mathrm{SiO}_{2}$ nanoparticle conditions changed the surface contact area with the low surface tension liquids. The lack of the hierarchical structure cannot maintain the air layer against oils. On the other hand, the appropriate $\mathrm{SiO}_{2}$ nanoparticle conditions which formed the hierarchical structure on the fabrics maintained air layer against low surface tension oils.

\subsection{Transparency of thin films}

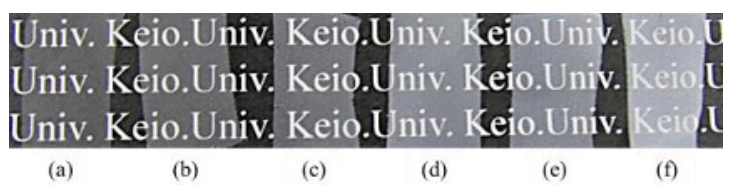

Figure 4. The optical image of spray-coated fabrics with different $\mathrm{SiO}_{2}$ nanoparticle concentration:

(a) $0 \mathrm{wt} \%$, (b) $0.25 \mathrm{wt} \%$,(c) $0.5 \mathrm{wt} \%$, (d) $0.75 \mathrm{wt} \%$, (e) $1.5 \mathrm{wt} \%$, (f) $3.0 \mathrm{wt} \%$

Figure 4 shows the optical images of spray-coated fabrics. As the increase of $\mathrm{SiO}_{2}$ nanoprarticle concentration, the transparencies of fabrics were decreased. The colors of fabrics became white with the increase of $\mathrm{SiO}_{2}$ nanoparticle concentration.

Figure 5 shows the parallel transparency and diffused light scattering percentage of spray-coated fabrics. As the $\mathrm{SiO}_{2}$ nanoparticle concentration increased from $0 \mathrm{wt} \%$, parallel transparency of thin films decreased. At the $\mathrm{SiO}_{2}$ nanoparticle concentration was $1.5 \mathrm{wt} \%$, the parallel transparency was $61.3 \%$. On the other hand, the 
percentages of diffused light drastically changed between $0.75 \mathrm{wt} \%$ and $1.5 \mathrm{wt} \%$. The diffused light percentage is based on the light scattering of fabrics morphology. This diffused light percentage change from $0.75 \mathrm{wt} \%$ to 1.5 wt $\%$ is supposed to relate the surface hierarchical structure on fabrics.

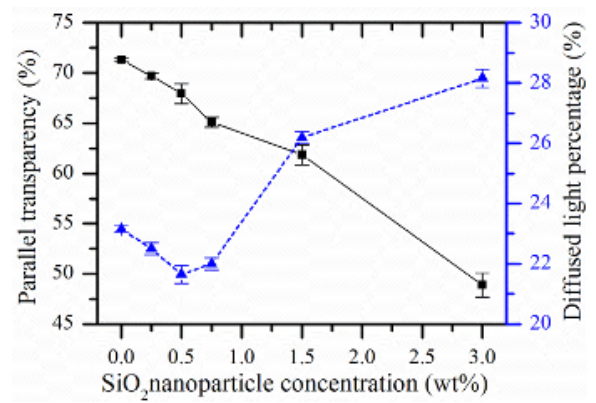

Figure 5. The parallel transparency and diffused light scattering percentage of spray-coated fabrics

Table 1. The relationships of transparency and the increased the diameters of fibrils

\begin{tabular}{ccccc}
\hline $\begin{array}{c}\mathrm{SiO}_{2} \\
\text { nanoparticle } \\
\text { concentration } \\
(\mathbf{w t} \%)\end{array}$ & $\begin{array}{c}\text { Parallel } \\
\text { transparency } \\
(\%)\end{array}$ & $\begin{array}{c}\text { Decrease of } \\
\text { transparency by } \\
\text { spray coating } \\
(\%)\end{array}$ & $\begin{array}{c}\text { Increase of } \\
\text { diameters of } \\
\text { fibrils } \\
(\mu \mathrm{m})\end{array}$ & $\begin{array}{c}\text { Ratio of fabrics } \\
\text { against } \\
\text { total surface area }\end{array}$ \\
\hline 0 & 71.6 & 0.2 & 0.2 & 28.4 \\
0.25 & 69.2 & 2.6 & 2.7 & 30.8 \\
0.50 & 67.7 & 4.1 & 4.5 & 32.3 \\
0.75 & 65.1 & 6.7 & 7.2 & 34.9 \\
1.5 & 61.1 & 10.7 & 11.7 & 38.9 \\
3.0 & 48.9 & 22.9 & 26.2 & 51.1 \\
\hline
\end{tabular}

The relationships of the parallel transparency and the geometrical calculation of coating fabrics are shown in Table 1. For the see-through structure, the parallel transparency is almost equal to the vacancy ratio of fabrics. Therefore, the increases of diameters of fibrils are calculated from geometrical relationship as follows:

$$
V=b^{2} /(a+b)^{2}
$$

where the $V$ is the vacancy ratio of fabrics, $a$ is the diameter of fibril and $b$ is the space distance between fibrils. This equation indicates that the increased diameters of fibrils are evaluated with the change of transparency. The diameters of superoleophobic fabric, sprayed condition of $1.5 \mathrm{wt} \% \mathrm{SiO}_{2}$, were raised at 11.7 $\mu \mathrm{m}$ from non-treated fabric. As a result, the diameters of nanoparticle structured fibrils are around $40 \mu \mathrm{m}$. The ratio of fabrics against total surface area is related to the contact area between air and solid in wettability. Although this value of the $\mathrm{SiO}_{2}$ nanoparticle $1.5 \mathrm{wt} \%$ condition was higher than that of $0 \mathrm{wt} \%$ or $0.5 \mathrm{wt} \%$, the liquid droplets didn't infiltrate the space of each fibrils because the surface contact area ratio was 0.1 in figure 3 . Cohen et.al. reported the liquid infiltration theory as the robustness factor in oleophobic region which depends on the space distance and its shape $[6,16]$. In our system, the shape of surface structure was also significant for enhancing oleophobicity.

As shown in Figure 6, compared with the result of Figure 3 which the spray-coated fabrics on the black paper with white textiles, this fabrics looks high transparency because of low contrast between color of the fabric and that of back ground in the picture. Since the diameters of each fabric were around $40 \mu \mathrm{m}$, each fabrics are hardly seen in this photo.

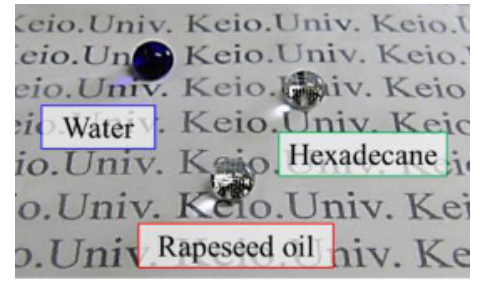

Figure 6. The optical image of water and oil droplets on the spray-coated fabrics. The three droplets stayed almost transparent fabric with superoleohobicity.

\section{Conclusion}

Newly fabricated see-through fabrics coated with the hierarchical particles structure show contact angles of $150^{\circ}$ with oils (rapeseed oil and hexadecane) and $61.3 \%$ of transparency. The hierarchical structure of nanoparticle on fabrics enhanced oleophobicity, and the scales of this hierarchical structure were over $10 \mu \mathrm{m}$. Considering the applicative possibility of see-through superoleophobic thin films, it has some of unique characters: free-standing, nearly invisible with appropriate contrast and durability of micro structure of fabrics.

\section{References}

1. C.R. Crick, I.P. Parkin, Chem. Eur. J. 163568 (2010)

2. X. Feng, L. Jiang, Adv. Mater. 18 3063(2006)

3. X. Li, D. Reinhoudt, M. Crego-Calama, Chem. Soc. Rev. 361350 (2007)

4. A.B.D. Cassie, S. Baxter, Trans. Faraday Soc. 40 546 (1944)

5. R.N. Wenzel, Ind. Eng. Chem. 28988 (1936)

6. W. Choi, A. Tuteja, S. Chhatre, J.M. Mabry, R.E. Cohen, G.H. McKinley, Adv. Mater. 212190 (2009)

7. A. Tuteja, W. Choi, M. Ma, J.M. Mabry, S.A. Mazzella, G.C. Rutledge, G.H. McKinley, R.E. Cohen, Science 3181618 (2007)

8. A. Steele, I. Bayer, E. Loth, Nano Lett. 9501 (2009)

9. H.Budunoglu, A.Yildirim, M.O. Guler, M.Bayindir ACS Appl. Mater. Interfaces 3539 (2011)

10. X.Deng, L.Mammen, Y.Zhao, P.Lellig, K.Müllen, C.Li , H.J.Butt, D.Vollmer Adv. Mater. 232962 (2011)

11. J. Zhang, S. Seeger, Angew. Chem. Int. Ed. 50 6652(2011)

12. R.Dufour, M. Harnois, Y. Coffinier, V. Thomy, R. Boukherroub, V. Senez, Langmuir 2617242 (2010)

13. W.Gaynor1, G.F. Burkhard, M.D. McGehee1, P.Peumans, Adv. Mater. 23 2905(2011)

14. M. Layani, S. Magdassi, J. Mater. Chem. 2115378 (2011)

15. B. Leng, Z. Shao, G. de With, W. Ming Langmuir 25, 2456 (2009)

16. S.S. Chhatre, W. Choi, A. Tuteja, K. Park, J.M. Mabry, G.H. McKinley, R.E. Cohen Langmuir 26 4027(2010) 